\title{
Intermittent preventive treatment for malaria and anaemia control in Tanzanian infants; the development and implementation of a public health strategy
}

\author{
Fatuma Manzi a,*, Joanna Schellenberg ${ }^{a, b}$, Yuna Hamis ${ }^{a}$, Adiel K. Mushi ${ }^{a}$, \\ Kizito Shirima a, Alex Mwitac, Azma Simba ${ }^{c}$, Neema Rusibamayilac, \\ Mary Kitambi ${ }^{c}$, Marcel Tanner ${ }^{d}$, Pedro Alonso ${ }^{e}$, Hassan Mshinda ${ }^{a}$, \\ David Schellenberg ${ }^{a, b}$
}

\author{
a Ifakara Health Institute (IHI), P.O. Box 78373, Dar es Salaam, Tanzania \\ ${ }^{\mathrm{b}}$ London School of Hygiene and Tropical Medicine, London, UK \\ c Ministry of Health, Dar es Salaam, Tanzania \\ d Swiss Tropical Institute, Basle, Switzerland \\ e Centre for International Health, Institut de Investigaciones Biomedicas August Pi I Sunyer (IDIBAPS), Barcelona, Spain
}

Received 4 October 2007; received in revised form 18 August 2008; accepted 18 August 2008 Available online 26 September 2008

\section{KEYWORDS \\ Malaria; \\ Child health; \\ Intermittent \\ preventive treatment in infants; \\ Expanded Programme on Immunization; \\ Health system; \\ Tanzania}

\begin{abstract}
Summary Minimizing the time between efficacy studies and public health action is important to maximize health gains. We report the rationale, development and implementation of a district-based strategy for the implementation of intermittent preventive treatment in infants (IPTi) for malaria and anaemia control in Tanzania. From the outset, a research team worked with staff from all levels of the health system to develop a public-health strategy that could continue to function once the research team withdrew. The IPTi strategy was then implemented by routine health services to ensure that IPTi behaviour-change communication materials were available in health facilities, that health workers were trained to administer and to document doses of IPTi, that the necessary drugs were available in facilities and that systems were in place for stock management and supervision. The strategy was integrated into existing systems as far as possible and well accepted by health staff. Time-and-motion studies documented that IPTi implementation took a median of 12.4 min (range 1.6-28.9) per nurse per vaccination clinic. The collaborative approach between researchers and health staff effectively translated research findings into a strategy fit for public health implementation.

(c) 2008 Royal Society of Tropical Medicine and Hygiene. Published by Elsevier Ltd. All rights reserved.
\end{abstract}

\footnotetext{
* Corresponding author. Present address: Mikocheni, Plot 463, Kiko Avenue, P.O. Box 78373, Dar es Salaam, Tanzania.

Tel.: +255 222 774756; fax: +255 222771714 .

E-mail addresses: manzif@yahoo.com, fmanzi@ihrdc.or.tz (F. Manzi).
}

0035-9203/\$ - see front matter @ 2008 Royal Society of Tropical Medicine and Hygiene. Published by Elsevier Ltd. All rights reserved. doi:10.1016/j.trstmh.2008.08.014 


\section{Introduction}

Malaria remains a major challenge in many developing countries, with recent estimates of the annual number of deaths directly due to malaria of over 800000 , mainly in children under 5 years living in Africa. ${ }^{1}$ Renewed focus and resources are being paid to malaria prevention and treatment in Africa, with major investment into the development and testing of antimalarial drugs and a malaria vaccine. However, there has been less investment in ensuring effective systems exist for the delivery of these new, and even existing, products. ${ }^{2,3}$

Intermittent preventive treatment in infants (IPTi) is a promising tool to fight malaria in children aged under 1 year. IPTi consists of the delivery of three treatment doses of an antimalarial drug alongside routine vaccinations. Most experience has been gained with sulfadoxine-pyrimethamine (SP), which has been licensed for use in children for over 30 years. Doses of IPTi are given as directly observed therapy (DOT) alongside routine vaccinations against DPT/OPV (at about 2-3 months of age) and measles (about 9 months of age). IPTi is given regardless of the presence of symptoms or parasitaemia. An initial study in Ifakara, southern Tanzania, showed that IPTi with SP was safe and reduced the incidence of clinical malaria and severe anaemia by at least $50 \%$ in the first year of life. ${ }^{4}$ In recent years clinical trials have been conducted In a range of other settings to explore the potential value of IPTi more broadly, ${ }^{5-10}$ and a research consortium is generating additional data to inform policy-makers. ${ }^{11}$ The new emphasis on the global research agenda to work on operational feasibility of interventions is a catalyst to undertaking effectiveness studies. ${ }^{12-16}$ In southern Tanzania, where initial safety and efficacy data already exists, it has been possible to address operational issues surrounding the deployment of IPTi through routine health systems.

The challenge of delivering efficacious tools to their target group is increasingly recognized. ${ }^{17}$ The translation of positive research results into policy and then public health action often follows a stuttering and uncertain path. For example, the first evidence of an impact of insecticidetreated mosquito nets (ITNs) on child survival was published in $1991,{ }^{12}$ and further evidence followed from a coordinated series of randomized trials. ${ }^{14,16,18-20}$ However, the 2002 African Malaria Report showed that less than 2\% of African children were using ITNs. ${ }^{21}$ The delivery of ITNs requires the establishment of a novel delivery strategy and is more complex than vaccination-linked interventions. However, even interventions delivered alongside the Expanded Programme on Immunization (EPI) vaccines may face problems: vitamin A supplementation at the time of vaccination against measles was made policy in Tanzania in 1987, yet coverage in the target group 12 years later was only $14 \%{ }^{22}$ Research into optimizing delivery strategies, exploring operational feasibility, acceptability, cost and cost-effectiveness under real-life, district-based programme conditions should accelerate the process of moving from efficacy to public health action.

This paper describes our approach to the development of an IPTi strategy that could be integrated into the Tanzanian EPI. The strategy involved development of an approach including behaviour-change communication messages and materials, a training curriculum for health workers, administrative systems for SP supply and accountability and systems to enable supervision and support at district and regional levels.

\section{Methods and results}

\subsection{Study area}

The IPTi strategy was developed for initial implementation in five districts in the Lindi (Lindi Rural, Nachingwea and Ruangwa) and Mtwara (Newala and Tandahimba) regions of southern Tanzania. These districts are part of the southern zone, which has the highest child mortality in Tanzania: the under-five mortality rate was reported as $153 / 1000$ live births in the ten-year period preceding the 2004/5 survey. ${ }^{23}$ The total population is around $900000 .{ }^{24}$ Described in detail elsewhere, ${ }^{25}$ the area is largely low lying with a broad mix of ethnic groups, although Swahili is widely spoken. Most people live in mud-walled, grass-roofed houses and depend on subsistence farming, fishing or small-scale trading for their livelihood. Most rural roads are unpaved, many becoming impassable during the rainy seasons. The public health system comprises a network of dispensaries, health centres and hospitals. Malaria is the leading public health problem according to regional health reports.

\subsection{The approach to strategy development}

We were keen to develop a strategy that could reasonably be expected to be implemented by the Ministry of Health $(\mathrm{MoH})$ on withdrawal of the research team. Hence, from the beginning the researchers worked in partnership with $\mathrm{MoH}$ policy-makers, managers and implementers at all levels, and involved a large number of in-country stakeholders. Figure 1 shows the approach to the development and implementation of IPTi, giving the main activities undertaken, timeline and stakeholders' involvement and interactions. The initial phase of sensitization and development of the strategy took approximately 1 year. However, the aim to minimize the time gap between demonstration of benefits and implementation in practice relates to policy and practice on a national scale. The policy process itself and the time it takes is beyond the scope of our paper.

At the national level we formed an ad-hoc 'IPTi Core Group', which steered the development of the strategy and lent political support. The group consisted of named, midlevel managers, who were more likely than senior managers to be able to attend meetings and were less likely to be replaced. The group comprised representatives from the national EPI programme, the National Malaria Control Programme (NMCP) and from the Integrated Management of Childhood Illnesses (IMCI) unit of Reproductive and Child Health (RCH) department of the MoH. WHO and UNICEF representatives were also invited to Core Group meetings. The Core Group guided the development of a training curriculum for front-line health workers, and training materials, and advised on who should be trained from each facility, where and by whom. They also provided a critical review of draft behaviour-change communication materials and took 


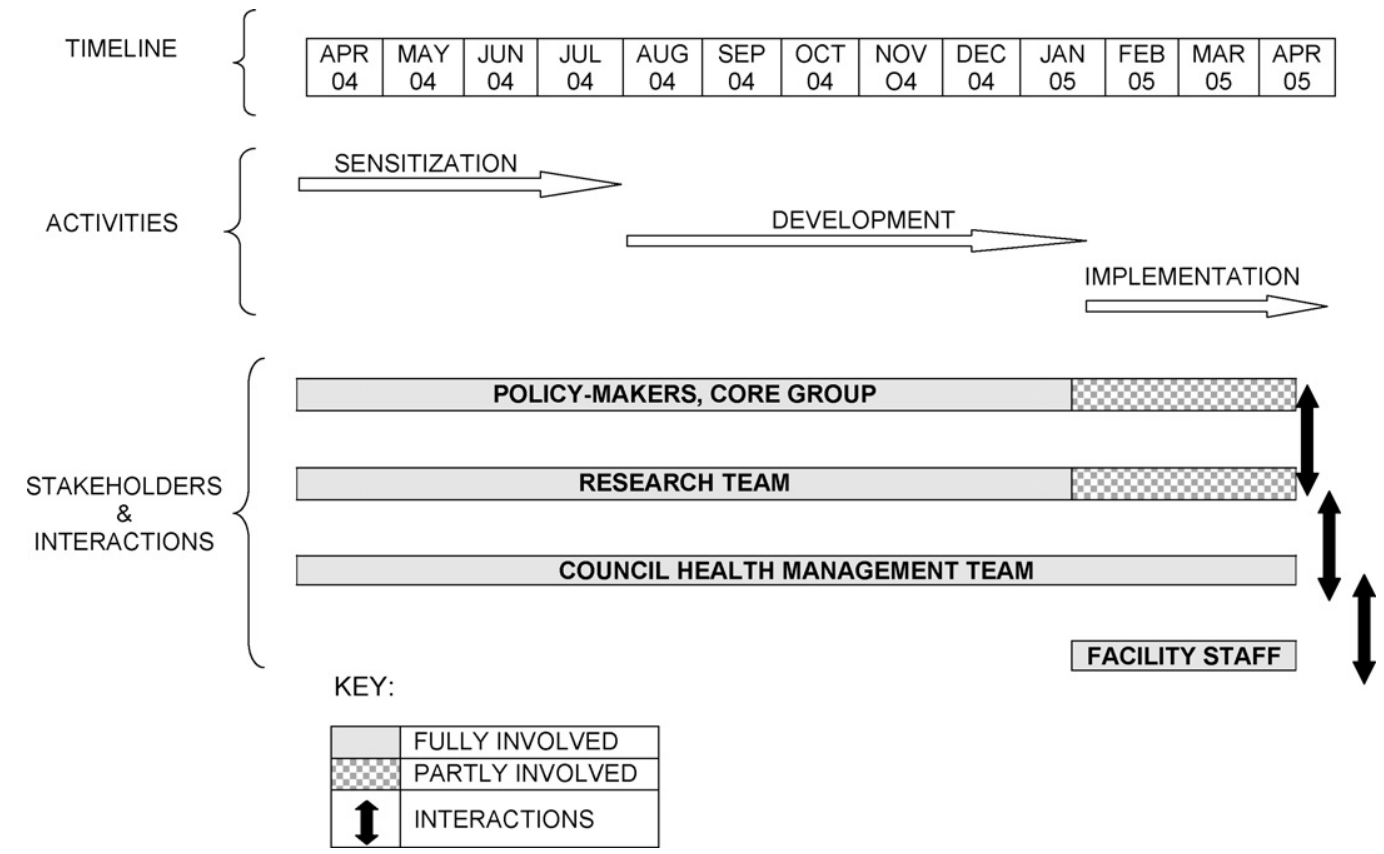

Figure 1 The approach to the development and implementation of intermittent preventive treatment in infants (IPTi) - the activities undertaken, timeline and stakeholders' involvement and interactions.

part in piloting and training of district-level trainers. The overall result was an IPTi strategy readily integrated into, and accepted by, the health system.

National-level stakeholder meetings were conducted regularly and involved additional staff from the EPI, NMCP and $\mathrm{RCH} / \mathrm{IMCl}, \mathrm{WHO}$ and UNICEF country offices, as well as staff from the Health Management and Information System, Medical Stores Department, Central Transport Unit of the $\mathrm{MoH}$, the Tanzania Food and Drug Authority, National Bureau of Statistics, National Institute for Medical Research, Muhimbili University College of Health Sciences and the Centre for Enhancement of Effective Malaria Interventions. Progress and plans, concerns and criticisms were shared with this group, and the IPTi strategy was further strengthened as a result. Although regional medical officers and $\mathrm{RCH}$ staff sometimes joined national stakeholder meetings, a series of planning and feedback meetings were held at regional and district levels during strategy development. These provided a wealth of useful, practical feedback on strategy development, communication and training materials and approaches to documentation and delivery of IPTi.

All through strategy development we considered only options that could be sustained on a public health scale, by existing front-line health staff, without ongoing inputs from the research team. For example, we did not use community sensitization and mobilization through street theatre or mass outreach campaigns because the costs are likely to be prohibitive in the context of routine implementation by the health system. Preparation for implementation involved two sets of activities. The first consisted of the development and testing of a brand name, messages of awareness-raising posters and a health-staff training programme, with relevant support materials. The second led to the development of management systems for IPTi, which were incorporated into the routine health system.

\subsection{Posters and training programme}

A rapid ethnographic study provided inputs for the behaviour-change communication strategy, including delivery channels, materials and a brand name for IPTi (A. Mushi et al., unpublished data). In brief, socio-cultural information was collected on local messages on perceptions of malaria and anaemia, vaccination clinics, attitudes to SP as a drug to prevent and treat malaria, and communication channels for health issues. A brand name for IPTi and draft materials were pre-tested in focus groups and in-depth interviews at village level, as well as in individual consultations with national, regional and district health staff as well as other stakeholders. The rapid ethnographic study revealed that mothers mostly learn about health issues from health workers and through informal discussions with other women. The existing posters at health facilities were seldom referred to by mothers. Nevertheless, we developed two posters promoting IPTi for use in health facilities, with the aim of guiding front-line health staff and mothers about key IPTi messages as well as providing a focus for health education to mothers attending vaccination clinic. The soliciting of comments on the draft materials was a useful mechanism to engage stakeholders at all levels.

We chose the brand name MKINGE (a Swahili word literally meaning (protect him or her') and the slogan MKINGE mtoto wako dhidi ya malaria ('protect your baby against malaria'). One of the posters showed a queue of happy women waiting for a nurse to give IPTi to their baby at a vaccination clinic. The two captions translated into 'Protect your baby from malaria' and 'MKINGE is a strategy to give SP to babies when they are given vaccines at 2, 3 and 9 months of age'. The second poster showed a healthy mother and her child in a rural setting and was captioned 'MKINGE reduces 
malaria and anaemia' and 'Many children have already used MKINGE'. The posters bore the logos of the key stakeholders, including the $\mathrm{MoH}$, and the Ifakara Health Research and Development Centre.

\subsection{Management tools and systems}

IPTi is administered three times during the first year of life, at the same time as vaccines delivered by the EPI system. It thus makes good sense to take advantage of the well-developed, tried and tested and widely understood EPI management systems. Forecasting the number of doses of SP for IPTi was based on the EPI tool used to forecast the number of doses of DPT vaccine, which is also administered three times in the first year of life. Although the research project supported implementation and delivered SP for IPTi to the district level, the Core Group planned how SP for IPTi would follow the same delivery channel from national to regional level as the syringes supplied for vaccination. This approach ensured that the correct number of SP doses were supplied but without making additional demands on the relatively expensive cold chain for vaccine supply. The required numbers of doses of SP would be delivered to regional vaccine stores in response to their requests. A similar 'pull' approach was employed from regional to district vaccine stores and to individual health facilities. Delivery to health facilities of SP for IPTi was thus linked to delivery of routine vaccines, subject to the same accountability controls and the responsibility of the District Cold Chain Officer. The minimum and maximum stock levels were set in the same way as for vaccines (a minimum of 1 month's supply and a maximum of 3 months' supply). At each facility the drugs were signed for in a ledger used for all other drug and vaccine stocks, and updated to show stock used each day. At facility level, the person in charge of the RCH clinic would be given responsibility for IPTi, including the safe storage of drugs. It was decided that IPTi drugs should not be in the care of the overall facility in-charge to ensure that SP for IPTi would be available even if the facility in-charge was away. Labelled plastic boxes were given to each facility for storage of SP for IPTi.

The administration of a dose of SP for IPTi was recorded in three places. Firstly, the routine tally sheet, which records the numbers of each vaccine administered, was edited to incorporate a section for IPTi. Secondly, the child's health card was also edited so that dates of IPTi doses could be recorded in the same area as the routine vaccinations. Thirdly, the register of children regularly attending the facility, which includes space to document some of the routine vaccinations, was also amended to enable recording of doses of IPTi. In practice, the tally sheet is used as the basis for a monthly summary of the numbers of doses of the different vaccines administered. A specific 'EPI monthly report' is completed in each facility and sent to the District Cold Chain Officer, whose responsibility is to complete a monthly summary report of all facilities in the district and forward to the Regional Cold Chain Officer, who completes and forwards to national level. By incorporating IPTi into each summary sheet, a system to account for IPTi, and to estimate coverage, was established.

\subsection{Implementation of the IPTi strategy}

The decentralized health system in Tanzania means that health service delivery is the responsibility of District Council Health Management Teams (CHMTs). As part of the IPTi strategy, the research team seconded from a CHMT an 'IPTi project implementation coordinator': a Clinical Officer with public health experience. In conjunction with CHMT members, he organized the training sessions, mobilized district and regional health staff for training and later supported implementation of the IPTi strategy in the five districts. Other project staff with direct experience of IPTi and development of other public health strategies, provided technical support. Implementation of IPTi was done in a phased approach, with health facility staff in half of all divisions (a division being an administrative level below a district) being selected randomly for training to implement IPTi. This cluster-randomized approach allowed robust evaluation of the IPTi strategy. Thus, staff from a randomly selected half of all health facilities were trained to implement IPTi in early 2005.

In an initial phase, lasting about 1 month, the IPTi strategy was piloted in two health facilities. After this, messages and materials were finalized and plans made for regional staff to train district health staff as trainers, and for the district staff in turn to train the front-line health staff as primary implementers. Materials developed to support training and implementation included an 'IPTi guideline' booklet, developed to guide the training of health workers and consisting of an overview of IPTi, a section with step-by-step instructions on how to administer and record doses of IPTi and a set of frequently asked questions. A laminated, A3sized job aid contained the step-by-step instructions and was intended for use in health facilities, either on the nurse's table or on a wall in the vaccination area. An initial stock of SP tablets for IPTi, posters, modified child health cards and tally sheets were also supplied during initial training, as were adhesive stickers to enable recording of IPTi doses on the child health cards of children who had already received health cards but who would become eligible for a dose of IPTi.

Piloting was started by a team formed by the project implementation coordinator, two other members of the project team, an NMCP representative, the Regional Reproductive and Child Health Coordinator from each of the two regions, one District Medical Officer, one District Reproductive and Child Health Coordinator, and one District Cold Chain Officer. This team reviewed the training guidelines and discussed operational issues such as the management and storage of IPTi supplies at district and facility levels. The majority of health facilities in the area had no supply of clean water, and after discussion with CHMT, it was decided to encourage health staff to purchase a socially marketed chemical purification liquid (WaterGuard, Mukwano Industries, Kampala, Uganda), and also to buy cups and spoons where necessary, using funds generated from user charges.

\subsection{Training of health staff}

One day of training for six staff from each CHMT was done in each region, led by the two Regional Reproductive and Child 
Health Coordinators with technical support from the IPTi project implementer, two other members of the research team and a member of the NMCP. From each district, the District Medical Officer, District Reproductive and Child Health Coordinator, District Cold Chain Officer, Pharmacist, Nursing Officer and malaria focal person attended the training. Regional medical officers, pharmacists and cold chain officers were also invited. Training included lectures, group discussions, practical work and a test of understanding. Following a general introduction a step-by-step explanation of how to administer and document IPTi was given. Before administering IPTi, health workers were reminded to check whether children had ever had an adverse reaction to any sulphur-containing drug such as co-trimoxazole or SP, and also whether they had received SP for malaria treatment in the previous 2 weeks, in order to avoid giving IPTi to those children. Other issues covered included how to order SP for IPTi, storage of SP, distribution and tracking within each district, supervision of IPTi activities and plans for training in each district. It was agreed that IPTi supervision would follow the same procedures as EPI vaccines, with district EPI staff (District Cold Chain Officer or District Reproductive and Child Health Coordinator) being responsible for delivery of supplies to each facility, checking stock, reviewing recordkeeping and supporting front-line health workers. Materials for training included the guideline booklet, job aid, posters, modified child health cards, stickers to record IPTi doses on the child health cards of children who already had cards, the modified vaccine tally sheets and stickers for modification of the village child health register. An initial stock of SP tablets for IPTi was also given to trainees.

In the weeks that followed, 115 front-line health staff from 61 dispensaries, health centres and hospitals attended the one-day training course. The training approach followed that used to train the trainers but with more emphasis on practical activities: why, when, how and to whom IPTi should be administered, storage, record-keeping and problem-solving. The training was led by three members of the CHMT (District Medical Officers, District Reproductive and Child Health Coordinators and District Cold Chain Officers) under the observation and technical support of the IPTi Project Implementation Coordinator. Two participants were invited from each health facility: the overall in-charge and the in-charge of the $\mathrm{RCH}$ section of the facility. The overall in-charge was included so that they would support their $\mathrm{RCH}$ staff to administer IPTi and would know to check before prescribing SP to a young child.

As part of the training, an initial supportive supervision visit was made to all participating health facilities within the following 2 months. District EPI staff and the IPTi Project Implementer reinforced the key messages of the one-day training session and helped to solve any problems. By October 2006, 92\% of facilities $(57 / 62)$ had started to use WaterGuard. A few of the front-line staff did not start implementation until after this visit, some waiting to start implementation at the beginning of a month, others wanting reassurance and benefiting from an on-site demonstration to reinforce the training on the new intervention. The main issues identified related to secure storage of the SP and adequate documentation.

After these initial visits, subsequent IPTi implementation management and support was fully integrated into the

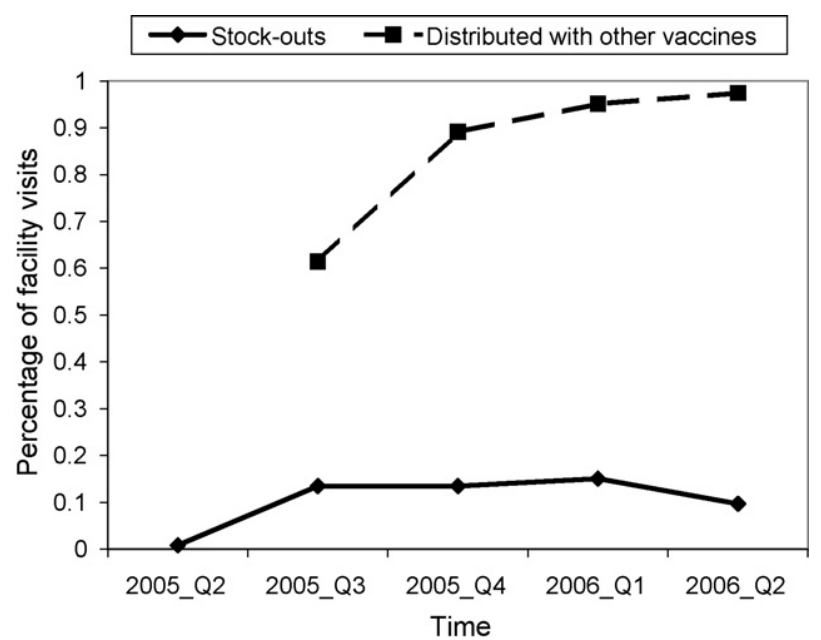

Figure 2 Intermittent preventive treatment in infants (IPTi) at health facilities, stock-outs and supplies of IPTi that were delivered to the facility at the same time as vaccines.

routine CHMT systems. The IPTi Project Implementer would sometimes join CHMT members during their routine monthly supervision visits in such a way that he visited most facilities every 2 months. Stock-outs of IPTi were a relatively common problem, found at roughly $13 \%$ of all visits. In the early months, vaccines were sometimes delivered without SP for IPTi, but as implementation continued the stock was increasingly distributed with vaccines (Figure 2). Coverage of each dose of IPTi at each facility each month was estimated using the number of children recorded on the tally sheet as the numerator and the established denominator based on the estimated population aged 0-11 months in the facility catchment's area (4\% of the population from the 2002 census, with adjustments for annual population growth, divided by 12 to get a monthly target population). Data were compiled at district level to generate estimates over multiple facilities or for larger periods of time; for example, in the first quarter of 2006, coverage of dose 3 of IPTi for implementing divisions of Newala District was 79\% (363/462) and for implementing divisions of Tandahimba District was $78 \%$ (594/764).

Nine months after the start of implementation we did qualitative and time-and-motion studies of RCH nurses delivering EPI vaccines and/or IPTi. The aim was to assess acceptability of IPTi to facility staff and to document the impact on time use of the new IPTi intervention. We did in-depth interviews with the nursing staff, exploring their expectations of IPTi implementation (for nurses in comparison divisions) or their actual experience of implementing IPTi (nurses in non-IPTi implementing, comparison divisions). Pairs of interviewers spent a week at each participant health facility. Towards the end of their stay, when the staff had grown used to the presence of the interviewers, the health workers were followed on a typical vaccine clinic day. Following positive earlier experiences with the use of personal digital assistants (PDAs) to capture health research data, ${ }^{26}$ we used PDAs to document the responses to direct questions and the time taken to give out IPTi health education and prepare and administer IPTi to infants, and the time taken to record delivery of the IPTi doses on immunization cards and 
HMIS books. This was done by means of a menu of nurses' activities on the PDA; when an activity was selected, the time was automatically recorded as the start time for that activity and the end time for the previous activity. The data were thus entered directly into Pendragon forms (Pendragon Software Corp., Libertyville, IL, USA) and synchronized into an Access database (Microsoft Corp., Redmond, WA, USA), where they were cleaned before analysis using Stata, version 8 (Stata Corp., College Station, TX, USA).

A total of 24 health facilities were sampled in the five study districts, selected to ensure balance in terms of type of health facility (dispensary or health centre), number of vaccinating staff and vaccine coverage between facilities in IPTi intervention and comparison areas. In comparison areas, when health workers were asked how difficult they thought it would be to implement IPTi, most said they were ready to implement, although a few were apprehensive about the anticipated increased work load and small number of staff. However, nurses in implementation areas said they had experienced no major difficulties in implementing IPTi. There were no reports of changes to the work schedules and only a few mentioned an obvious increase in the time spent on documentation. Among the 12 intervention facilities, nine had vaccination days with children eligible for IPTi. RCH nurses spent a median of $12.4 \mathrm{~min}$ ( $n=9$, range 1.6-28.9) on IPTi delivery and dose-recording on a typical vaccine clinic day. There was a median of more than $2 \mathrm{~h}$ non-productive time among nurses on these vaccine clinic days in both implementing and comparison facilities.

\subsection{Financial costs}

The study in southern Tanzania included tracking of health system and other costs of developing the strategy and maintaining routine implementation of the strategy in five districts, which are described elsewhere. ${ }^{27}$ Briefly, the estimated financial cost to start up and run IPTi in the whole of Tanzania in 2005 was US\$1,486,284. Start-up costs of US\$36363 were incurred at the national level, mainly on the development of behaviour-change communication materials, stakeholders' meetings and other consultations. The annual running cost at national level for intervention management and monitoring and drug purchase was estimated at US\$459096.

\section{Discussion}

Under the auspices of the IPTi Consortium ${ }^{11}$ and other groups, IPTi safety and efficacy studies have been completed or are ongoing in Ghana, Gabon, Mozambique, Kenya, Tanzania and Papua New Guinea (PNG), and large-scale, multi-district implementation of IPTi is ongoing in six countries under programmes run by UNICEF. The end product will be a detailed safety profile and an overview of the efficacy of IPTi from a range of epidemiological and geographic settings. Furthermore, the Consortium will have generated experience in the development and operationalization of IPTi strategies in a variety of health systems. Hence, it will be possible to make an evidence-based policy decision and then, if the recommendation is to push forward with IPTi, to facilitate rapid, large-scale implementation and public health action.

We have described the development and early implementation of an IPTi strategy within the context of a large-scale operational research programme in southern Tanzania. Broad institutional and political support were garnered through stakeholders meetings at various levels and helped to assure the integration of the IPTi strategy into existing service delivery. ${ }^{28}$ The Core Group was an efficient mechanism to develop a robust strategy by accessing the advice of key stakeholders on the development and finetuning of the delivery system. The establishment of this group helped maintain awareness and involvement in the process of strategy development, which has previously been shown to be vital in determining the longer-term utility of a health strategy. ${ }^{29}$ Researchers worked in partnership with $\mathrm{MoH}$ representatives to deliver a new intervention over a large area in a way that could readily be scaled up nationally. In so doing, we have tried to translate research findings into something that could be applied as public health action. ${ }^{30}$ The study had an external clinical monitor for quality assurance. Among the lessons learned in this process are that considerable time is needed to prepare for public health action, in addition to evidence of benefit, a collaborative approach and adequate financial resources.

Delivery of IPTi through well-established routine systems such as EPI also increases the chance of long-term sustainability, saves on implementation costs and should make the approach relatively attractive to policy-makers. The initial activities described here have generated information on costs and experience with the issues surrounding drug supply, training, supervision and development of implementation guidelines that could speed up implementation at country level. The EPI is unparalleled in its ability to deliver preventive interventions to young children in Africa. In its 30 year existence the EPI has developed into a well-respected vertical programme at national level, which is fully integrated with other health services at district level and at the point of service delivery. Coverage figures in Tanzania show that $71 \%$ of all children aged $12-23$ months have received all their routine vaccines by the age of 12 months, and $96 \%$ of children have received at least one vaccine. ${ }^{23}$

Our coverage estimates are based on routine reporting and should be treated with the caution normally associated with such estimates, as both numerator and denominator are prone to errors. The number of children who have received the intervention is recorded on a simple tally-sheet, and with this approach it is easy to record the same child twice and to forget to record other children. The target populations are also prone to errors due to temporary or permanent in and out migration, seasonal variation in births and difficulty defining target populations. Nevertheless, routine estimates of vaccine coverage match relatively well with estimates derived from a household survey in the same area. $^{25}$

Our IPTi strategy is embedded within a research study, and hence the conditions under which IPTi is implemented are not exactly as they would be in a national programme. IPTi is not implemented in all health facilities, but in a randomly selected half of the divisions. Although this detracts from the 'real-life' nature of the implementation it adds considerable strength to the evaluation. The full-time IPTi 
Project Implementer accompanied CHMT members on visits to health facilities about every 2 months, probably increasing the frequency and focus of IPTi-related activities during routine supervision visits. This may have increased IPTi coverage beyond that gained by a national programme, although follow-up visits were in the context of routine supervision by CHMT members and the approach mirrored routine implementation on a national scale.

After one full year of implementation, IPTi was nearly always distributed from district to facility level at the same time as routine vaccines, suggesting that district health staff had integrated IPTi into their usual routine. However, stockouts of IPTi remained relatively common. For example, 10\% of health facilities experienced at least one stock-out in the second quarter of 2006. Although this is of concern, it should be noted that stock-outs of routine vaccines were at least as common. For example, a health facility survey done in the study area in 2004 found that only $77 \%$ (91/118) of all facilities had all vaccines in stock on the day of the survey. ${ }^{25}$ Nevertheless, as most stock-outs were of short duration, overall vaccine coverage was greater than $80 \%$ for DPT and polio.

Staff in facilities where IPTi was not available expected to be able to implement an IPTi strategy, although some expressed reservations about the increased workload. However, no staff involved in IPTi implementation reported having to increase the number of vaccination days per week or reorganization of clinic staff to accommodate the increased workload. We observed IPTi-related activities taking a median time of less than $15 \mathrm{~min}$ per nurse per vaccination day, and there was no evidence of health worker time constraining the delivery of IPTi or other health interventions. We were surprised to find that vaccinating nurses had a median of over $2 \mathrm{~h}$ non-productive time per vaccination clinic day, although this is similar to other facility-based time-and-motion studies in Tanzania and elsewhere. ${ }^{31}$ Hence, IPTi could be rolled out in an integrated manner throughout the country by the health workers who are already available. It is important to note that, although human resources are a major problem in Tanzania and other developing countries, many aspects of health service delivery could be improved by enhanced time management and supportive supervision at facility level.

The overall purpose of the southern Tanzania IPTi effectiveness study was to prepare for prompt public health action when and if a positive policy recommendation is made. Policy recommendations are not made on the basis of research evidence alone, but rest on other issues as well. ${ }^{15,29,32}$ What are the consequences of an IPTi policy recommendation being delayed or not being made at all? In this case implementation would stop, and the lessons described here would have relevance for the future, for development of other public health strategies.

In undertaking this work, there were some negative experiences that need to be documented. Working with government employees at national, regional and district level, the research team found some individuals did not prioritize work related to IPTi, possibly because they felt they should have been paid extra to do it. At service delivery level, some staff who had received on-the-job training were not ready to administer IPTi if a colleague who had attended formal IPTi training was out of the working station. This reluctance was probably due to envy of formal trainees, who had received a travel allowance to attend the training. Supervision was an additional challenge, with infrequent visits, which were rarely supportive. These problems were tackled through communication and positive feedback for the purpose of bringing productive changes in public health delivery.

In conclusion, this study has generated useful experience on the development of a strategy for the routine delivery of IPTi. We found that combining researchers and programme implementers into a single Core Group enabled the development of a practical strategy that was readily implemented at facility and district level. The researchers led strategy development, and made the major investment in this respect, thus helping to translate research results into a strategy that could be implemented as a public health programme. We believe this partnership to be novel and hope that our experience can contribute to the design of delivery strategies, not only for IPTi but also for taking to scale other child health interventions.

Authors' contributions: FM and JS conceived the study; FM, JS and DS participated in the design of the study; FM, JS, $\mathrm{YH}, \mathrm{AKM}, \mathrm{KS}, \mathrm{AM}, \mathrm{AS}, \mathrm{NR}$ and MK participated in data analysis and interpretation; MT, PA and HM provided technical support; DS coordinated the study, data analysis and interpretation; FM and JS wrote the manuscript. All authors read, commented on and approved the final manuscript. FM and JAS are guarantors of the paper.

Acknowledgements: The authors express their sincere gratitude to all district health staff of Lindi Rural, Nachingwea, Ruangwa, Tandahimba and Newala, where we conducted this study. We also thank the regional medical officers of Lindi - Dr Ally Mohamed and Mtwara - and Dr Sylvester Budeba for their support during the course of the project. Further thanks go to the WHO country malaria coordinator and health staff at UNICEF for their participation in the IPTi core group and stakeholders' meetings. Finally, we thank Mwifadhi Mrisho for participation in the project development, and Shekha Nasser and Adeline Nderumaki for supporting IPTi project activities.

Funding: The study received funding from the Bill and Melinda Gates Foundation through the Intermittent Preventive Treatment of malaria in infants (IPTi) Consortium.

\section{Conflicts of interests: None declared.}

Ethical approval: The study was approved by the institutional review committees of the Ifakara Health Research and Development Centre (IHRDC), Dar es Salaam, Tanzania; National Medical Research Coordinating Committee of Tanzania; and London School of Hygiene and Tropical Medicine, London, UK. All study subjects gave informed consent to the work.

\section{References}

1. Rowe AK, Rowe SY, Snow RW, Korenromp EL, Schellenberg $\mathrm{JR}$, Stein $\mathrm{C}$, et al. The burden of malaria mortality among 
African children in the year 2000. Int J Epidemiol 2006;35: 691-704.

2. Jones G, Steketee RW, Black RE, Bhutta ZA, Morris SS. How many child deaths can we prevent this year? Lancet 2003;362:65-71.

3. Leroy JL, Habicht JP, Pelto G, Bertozzi SM. Current priorities in health research funding and lack of impact on the number of child deaths per year. Am J Public Health 2007;97:219-23.

4. Schellenberg D, Menendez C, Kahigwa E, Aponte J, Vidal $J$, Tanner $M$, et al. Intermittent treatment for malaria and anaemia control at time of routine vaccinations in Tanzanian infants: a randomised, placebo-controlled trial. Lancet 2001;357:1471-7.

5. Chandramohan D, Owusu-Agyei S, Carneiro I, Awine T, Amponsa-Achiano K, Mensah N, et al. Cluster randomised trial of intermittent preventive treatment for malaria in infants in area of high, seasonal transmission in Ghana. $\mathrm{Br}$ Med $\mathrm{J}$ 2005;331:727-33.

6. Grobusch MP, Lell B, Schwarz NG, Gabor J, Dornemann J, Potschke $M$, et al. Intermittent preventive treatment against malaria in infants in Gabon-a randomized, double-blind, placebo-controlled trial. J Infect Dis 2007;196:1595-602.

7. Kobbe R, Kreuzberg C, Adjei S, Thompson B, Langefeld I, Thompson PA, et al. A randomized controlled trial of extended intermittent preventive antimalarial treatment in infants. Clin Infect Dis 2007;45:16-25.

8. Macete E, Aide P, Aponte JJ, Sanz S, Mandomando I, Espasa M, et al. Intermittent preventive treatment for malaria contro administered at the time of routine vaccinations in mozambican infants: a randomized, placebo-controlled trial. $J$ Infect Dis 2006; 194:276-85.

9. Massaga JJ, Kitua AY, Lemnge MM, Akida JA, Malle LN, Ronn $A M$, et al. Effect of intermittent treatment with amodiaquine on anaemia and malarial fevers in infants in Tanzania: a randomised placebo-controlled trial. Lancet 2003;361: 1853-60.

10. Mockenhaupt FP, Reither K, Zanger P, Roepcke F, Danquah I, Saad E, et al. Intermittent preventive treatment in infants as a means of malaria control: a randomized, double-blind, placebo-controlled trial in northern Ghana. Antimicrob Agents Chemother 2007;51:3273-81.

11. ipti-malaria.org. Barcelona: The IPTi Consortium [updated 1 August 2008]. http://www.ipti-malaria.org [accessed 7 August 2008].

12. Alonso PL, Lindsay SW, Armstrong JR, Conteh M, Hill AG, David $\mathrm{PH}$, et al. The effect of insecticide-treated bed nets on mortality of Gambian children. Lancet 1991;337: $1499-502$.

13. Armstrong Schellenberg JR, Adam $T$, Mshinda $H$, Masanja $H$, Kabadi G, Mukasa O, et al. Effectiveness and cost of facilitybased Integrated Management of Childhood Illness (IMCI) in Tanzania. Lancet 2004;364:1583-94.

14. Binka FN, Kubaje A, Adjuik M, Williams LA, Lengeler C, Maude $\mathrm{GH}$, et al. Impact of permethrin impregnated bednets on child mortality in Kassena-Nankana district, Ghana: a randomized controlled trial. Trop Med Int Health 1996;1:147-54.

15. Crawley J, Hill J, Yartey J, Robalo M, Serufilira A, Ba-Nguz A, et al. From evidence to action? Challenges to policy change and programme delivery for malaria in pregnancy. Lancet Infect Dis 2007; 7:145-55.

16. D'Alessandro U, Olaleye B, Langerock P, Bennett S, Cham K, Cham B, et al. The Gambian National Impregnated Bed Net Programme: evaluation of effectiveness by means of case-control studies. Trans R Soc Trop Med Hyg 1997;91:638-42.

17. Victora CG, Hanson K, Bryce J, Vaughan JP. Achieving universal coverage with health interventions. Lancet 2004;364:1541-8.

18. Habluetzel A, Cuzin N, Diallo DA, Nebie I, Belem S, Cousens $\mathrm{SN}$, et al. Insecticide-treated curtains reduce the prevalence and intensity of malaria infection in Burkina Faso. Trop Med Int Health 1999;4:557-64.

19. Lengeler C. Insecticide-treated bednets and curtains for preventing malaria. Cochrane Database Syst Rev 2000;2:CD000363.

20. Nevill CG, Some ES, Mung'ala VO, Mutemi W, New L, Marsh K, et al. Insecticide-treated bednets reduce mortality and severe morbidity from malaria among children on the Kenyan coast. Trop Med Int Health 1996;1:139-46.

21. WHO. The African Malaria Report. Geneva: World Health Organization; 2003. http://www.rbm.who.int/amd2003/amr2003/ ch5.htm [accessed 7 August 2008].

22. National Bureau of Statistics and ORC Macro. Tanzania reproductive and child health survey 1999. Calverton, MD: NBS and Macro International; 2000.

23. National Bureau of Statistics. Tanzania Demographic and Health Survey 2004-2005. Dar es Salaam: National Bureau of Statistics Tanzania; 2005. http://www.nbs.go.tz/DHS [accessed 7 August 2008].

24. Tanzania National Website. 2002 Population and Housing Census. Dar es Salaam: National Bureau of Statistics; 2003. http://www.tanzania.go.tz/census [accessed 7 August 2008].

25. Armstrong Schellenberg JR, Mrisho M, Manzi F, Shirima K, Mbuya C, Mushi AK, et al. Health and survival of young children in southern Tanzania. BMC Public Health 2008;8:194.

26. Shirima K, Mukasa O, Schellenberg JA, Manzi F, John D, Mushi $A$, et al. The use of personal digital assistants for data entry at the point of collection in a large household survey in southern Tanzania. Emerg Themes Epidemiol 2007;4:5.

27. Manzi F, Hutton G, Schellenberg J, Tanner M, Alonso P, Mshinda $\mathrm{H}$, et al. From strategy development to routine implementation: the cost of intermittent preventive treatment in infants for malaria control. BMC Health Serv Res 2008;8:165.

28. Walley J, Khan MA, Shah SK, Witter S, Wei X. How to get research into practice: first get practice into research. Bull World Health Organ 2007;85:424.

29. Williams HA, Durrheim D, Shretta R. The process of changing national malaria treatment policy: lessons from country-level studies. Health Policy Plan 2004;19:356-70.

30. Davis $P$, Howden-Chapman $P$. Translating research findings into health policy. Soc Sci Med 1996;43:865-72.

31. Adam T, Manzi F, Schellenberg JA, Mgalula L, de Savigny D, Evans DB. Does the integrated management of childhood illness cost more than routine care? Results from the United Republic of Tanzania. Bull World Health Organ 2005;83:369-77.

32. Agyepong IA, Adjei S. Public social policy development and implementation: a case study of the Ghana National Health Insurance scheme. Health Policy Plan 2008;23:150-60. 